\title{
Configuration synthesis of generalized deployable units via group theory
}

\author{
Tuanjie $\mathrm{Li}^{1}$, Jie Jiang ${ }^{2}$, Hangjia Dong ${ }^{1}$, and Lei Zhang ${ }^{1}$ \\ ${ }^{1}$ School of Electromechanical Engineering, Xidian University, Xi' an, 710071, China \\ ${ }^{2}$ Engineering College, Honghe University, Mengzi, 661100, China \\ Correspondence to: Tuanjie Li (tjli888@126.com)
}

Received: 2 April 2016 - Revised: 28 May 2016 - Accepted: 9 September 2016 - Published: 15 September 2016

\begin{abstract}
The generalized deployable mechanism is composed of generalized links and generalized kinematic pairs. The generalized links include the flexible members, springs, and cables etc. The generalized kinematic pairs include the preloaded kinematic pairs and flexible hinges etc. The generalized deployable mechanism consists of generalized deployable units. Based on group theory, this paper presents a brief and effective configuration synthesis method for generalized deployable units which is composed of generalized links and generalized kinematic pairs expressed by group. The permutation group is used to obtain all the permutation types of generalized kinematic pairs and generalized links. The configuration matrix of generalized deployable unit is established through combining permutation group, and the topological configurations of generalized deployable units are created. The combining rules of groups are developed. The method of isomorphism detection is used to ensure the uniqueness of configurations. The configurations of generalized deployable units including four and six generalized links are generated respectively.
\end{abstract}

\section{Introduction}

The deployable mechanism is proposed by Pinero in the 1960s (Pinero, 1962). Deployable mechanisms, also named as deployable structures (Pellegrino, 2001), can vary their shape automatically from a compact, packaged configuration to an expanded, operational configuration. The first properly engineered deployable structures were used as stabilization booms on early spacecraft. Later on, more complex structures were devised for solar arrays, communication reflectors and telescopes (Li, 2014; Tibert, 2002; Mruthyunjaya, 2003). In other fields there have been a variety of developments, including retractable roofs for stadia, foldable components for cars, portable structures for temporary shelters and exhibition displays. Obviously, the requirements that have to be met by a deployable mechanism in its operational configuration (e.g. providing shelter from rain, in the case of umbrella, or forming an accurate reflective surface, in the case of a deployable reflector antenna for telecommunications) are different from the requirements in the package configuration (usually, this should be as small as possible). But an essen- tial requirement is that the deployment and retraction transformation process should be possible without any damage, and should be autonomous and reliable (Pellegrino, 2001). For this purpose, the deployable mechanisms are composed of same deployable units due to modular design. The deployable and foldable transformation of deployable units depends on the elastic deformation of springs, flexible components or cable driven. Thus, the deployable unit not only includes the traditional rigid links and kinematic pairs, but also includes the flexible components, springs, cables and the preloaded kinematic pairs and flexible hinges etc, and thus defined as the generalized deployable unit which is composed of generalized links and generalized kinematic pairs. The generalized links include flexible members, springs, and cables etc. The generalized kinematic pairs include preloaded kinematic pairs and flexible hinges etc.

With the development of deployable mechanisms, it is possible to evolve new configurations that meet the requirements of engineering applications. The configuration synthesis is the effective method to address the key problem. The configuration synthesis of traditional mechanisms has 
been one of hotspot issues. The early configuration synthesis mainly depended on the experience and inspiration. Until the 1960 s, the topological graph theory was introduced to describe the mechanisms and lots of configuration synthesis methods were advanced (Davies, 1966). For the deployable mechanisms, Gantes (1991) investigated the geometric modeling and design methodology of deployable structures featuring stable and stress-free states in both the deployed and the collapsed configurations. Warnaar and Chew (1995a, b) studied the generation of deployable truss modules with the aid of graph theory. Chen and You (2009) conducted lots of researches in the field of monocyclic constraint mechanisms on the basis of Bennett and Myard mechanisms, and generated some new space deployable mechanisms. Ding et al. (2013) proposed a novel deployable mechanism based on polyhedral linkages, and discussed the mobility and the singularities. Lu et al. (2014) proposed a family of novel deployable prism mechanisms based on the Hoekens straight-line linkages. Lu et al. (2015) researched a network of type III Bricard linkages, and performed two methods of connecting linkages. As an expansion of traditional deployable mechanisms consisted of rigid links and kinematic pairs, the generalized deployable mechanism, which is composed of generalized links and generalized kinematic pairs, is a system that can vary their shape automatically from a compact, packaged configuration to an expanded, operational configuration. The generalized links, as the expansion of the traditional rigid links, contain all members which are capable to transform motions and forces, including the flexible members, springs, and cables etc. The generalized kinematic pairs, as the expansion of the traditional pairs, contain all kinematic pairs which connect different links and offer motion constraints, including not only traditional prismatic pairs and revolute pairs but also preloaded prismatic pairs, preloaded revolute pairs, flexible hinges etc. The combination of different generalized links and generalized kinematic pairs may generate all sorts of configurations with different performances. The structural complexity of generalized deployable mechanisms results in that the topological representation and configuration synthesis cannot be addressed by the existing methods. Proceeding from the generalized links and generalized kinematic pairs, we develop the configuration synthesis of generalized deployable units based on the finite group theory. The configurations of generalized deployable units including four and six generalized links are created.

\section{Graphical representation and configuration matrix of generalized deployable units}

The generalized deployable units include cables, flexible components, and preloaded kinematic pairs etc. The adjacent matrix of graph theory is often used to describe topological relationship between links and kinematic pairs. Thus, the configuration matrix of generalized deployable units is

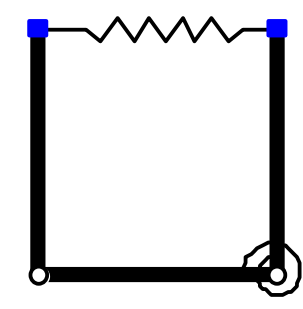

Figure 1. The four-link generalized deployable unit.

defined to describe the topological relationship between generalized links and generalized kinematic pairs. The diagonal elements in configuration matrix represent the type of generalized links, and the nondiagonal elements represent the type of generalized kinematic pairs. The weighted values in the configuration matrix are defined to express the types of generalized links and generalized kinematic pairs, as shown in Tables 1 and 2 .

In order to express the relation between the generalized links and generalized kinematic pairs, the configuration matrix is introduced to describe the generalized deployable units. The configuration matrix and the generalized deployable unit are one to one correspondence. The diagonal elements of configuration matrix represent generalized links, and other elements represent the relation between two generalized links. We have

$\mathbf{A}=\left(a_{i j}\right)_{n \times n}$,

where, $\mathbf{A}$ is the configuration matrix of a generalized deployable unit, $n$ is the number of generalized links, and

$a_{i j}= \begin{cases}w_{1} & \begin{array}{l}\text { If } i \neq j, \text { the weighted value of generalized } \\ \text { kinematic pair connecting two links. }\end{array} \\ w_{2} & \text { If } i=j, \text { the weighted value of generalized link. } \\ 0 & \text { Otherwise. }\end{cases}$

For example, a four-link generalized deployable unit is shown in Fig. 1, its configuration matrix is

$\mathbf{A}=\left[\begin{array}{llll}3 & 9 & 0 & 9 \\ 9 & 1 & 7 & 0 \\ 0 & 7 & 1 & 5 \\ 9 & 0 & 5 & 1\end{array}\right]$

\section{Configuration synthesis method}

The basic idea of configuration synthesis method is the link replacement in the traditional kinematic chains or frame structures. The configurations of traditional kinematic chains or frame structures are called as the initial configurations, and the corresponding matrix is called as the initial matrix. The main diagonal elements of initial matrix constitute a vector called as the links generator $a$. The non-zero elements 
Table 1. Types of generalized links.

\begin{tabular}{lcc}
\hline Generalized links & Weighted values $\left(w_{2}\right)$ & Graphical representation \\
\hline Rigid link & 1 & - \\
Flexible link & 2 & $-1 m$ - \\
Spring & 3 & \\
Cable & 4 & \\
\hline
\end{tabular}

Table 2. Types of generalized kinematic pairs.

\begin{tabular}{lcc}
\hline Generalized kinematic pairs & Weighted values $\left(w_{1}\right)$ & Graphical representation \\
\hline Revolute pair & 5 & 7 \\
Preloaded hinge & 8 & \\
Flexible hinge & 9 & \\
Fixed joint & & \\
\hline
\end{tabular}

of non-main-diagonal elements of initial matrix constitute a vector called as the kinematic pairs generator $b$. The vector groups of links and kinematic pairs are obtained from their generators by the defined generation relation. The matrix groups of links and kinematic pairs are established by the defined mapping relation. The configuration matrix groups are generated by combining the matrix groups of links and kinematic pairs. The generalized deployable units are created after removing the unreasonable configurations such as the locally rigid configurations, the unreasonable stress configurations and isomorphism configurations.

\subsection{Vector groups of links and kinematic pairs}

The vector groups of links and kinematic pairs are established by the defined generation relation for the generators. All the elements of link generator are 1, which means all the links are rigid. All the elements of kinematic pair generator are 5, which means all the kinematic pairs are revolute pairs. The generation relation of links is defined as follows.

$\varphi(i, h)=[1 \rightarrow i, h], \quad i=1,2,3,4$,

where, $i$ denotes as the generation relation number and the weighted values of links. The $h$ stands for the $h$ th element in the generator. Equation (2) transforms the weighted value of the $h$ th element in the generator from 1 (rigid link) to $i$ (other type).

Similarly, the generation relation of kinematic pairs is defined as follows.

$\psi\left(i_{Y}, h\right)=\left[5 \rightarrow i_{Y}, h\right], \quad i_{Y}=5,7,8,9$,

where, $i_{Y}$ represents the generation relation number and the weighted value of kinematic pairs.
For example, the generator $a=[1,1,1]$ means that all the links are rigid. The operation of $\varphi(2,4) \rightarrow a$ means the generation relation $\varphi(2,4)=[1 \rightarrow 2,4]$ is applied to $a$, and $a$ is then transformed into [1, 1, 2]. That is, the four rigid links are transformed into three rigid links and one flexible link.

The vector subgroups of links and kinematic pairs can be obtained while the generation relations are operated for the generators which is realized with a recursive function. The vector subgroups of links are generated as follows:

$$
\begin{aligned}
& \boldsymbol{G}_{1}=\varphi(i, 1) \rightarrow a \\
& \boldsymbol{G}_{k}=\varphi(i, k) \rightarrow G_{k-1}(h), k>1,
\end{aligned}
$$

where $k$ is the subgroup number.

The vector subgroups of kinematic pairs are generated as follows.

$$
\begin{aligned}
& \boldsymbol{Y}_{1}=\psi\left(i_{Y}, 1\right) \rightarrow b \\
& \boldsymbol{Y}_{k}=\psi\left(i_{Y}, k\right) \rightarrow Y_{k-1}(h), k \gg 1
\end{aligned}
$$

A series of subgroups of links and kinematic pairs are obtained in accordance with the above recursive functions. Every subgroup is a part of configurations, so all the configurations are the union of subgroups. The same configuration may exist in the different subgroups, which can be found by the intersection of subgroups. Thus, the vector groups of links and kinematic pairs are derived respectively as follows.

$$
\begin{gathered}
\boldsymbol{G}_{\mathrm{s}}=\bigcup_{k=1}^{m} G_{k}-\bigcup_{\ell=1, \delta=1, \ell \neq \delta}^{m(m-1)}\left\{G_{\delta} \bigcap G_{\ell}\right\} \\
\boldsymbol{Y}_{\mathrm{s}}=\bigcup_{k=1}^{n} Y_{k}-\bigcup_{\ell=1, \delta=1, \ell \neq \delta}^{n(n-1)}\left\{Y_{\delta} \bigcap Y_{\ell}\right\},
\end{gathered}
$$




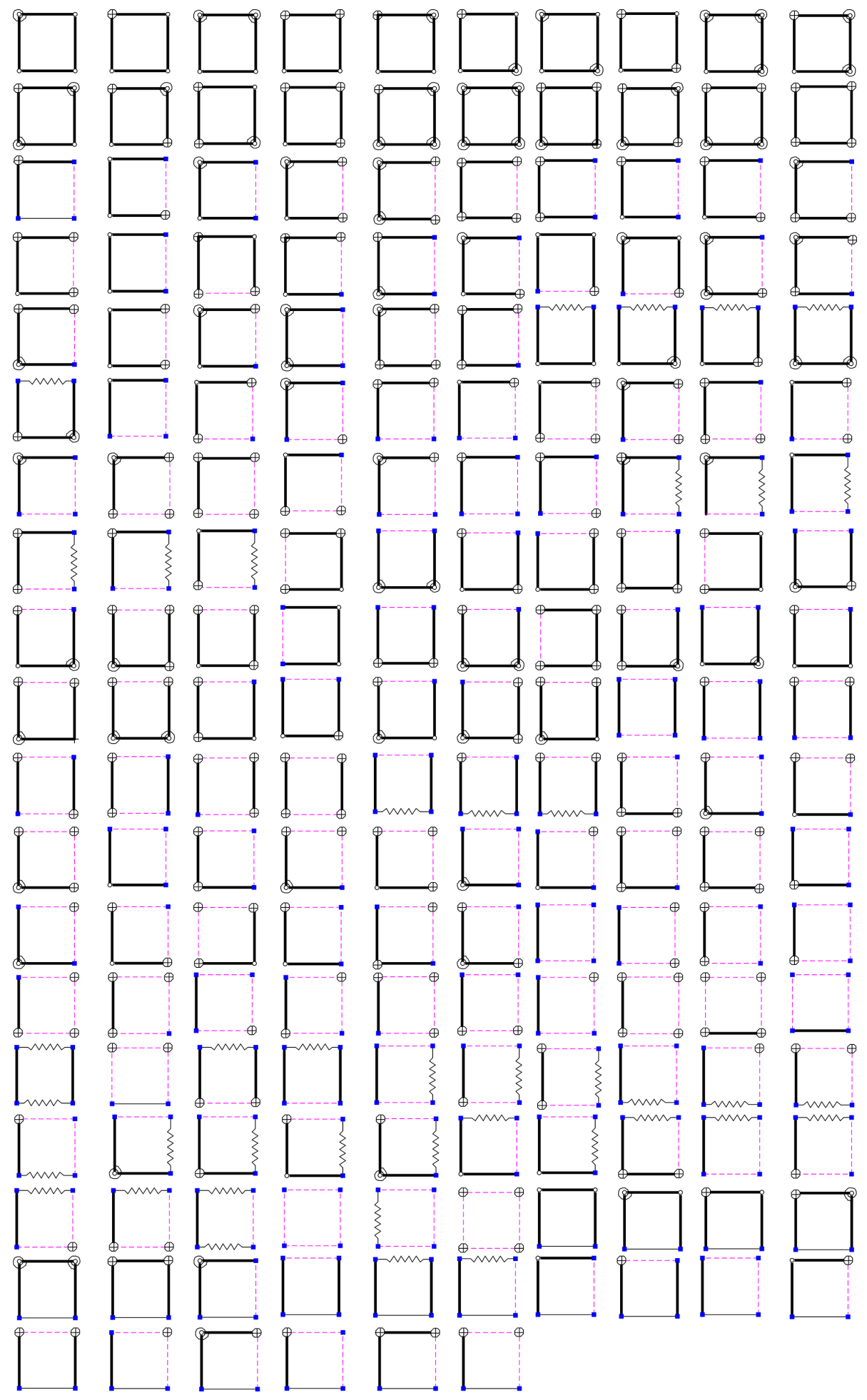

Figure 2. Configurations of 4-link generalized deployable unit.

where, $m$ denotes the number of link subgroups, and $n$ means the number of kinematic pair subgroups.

The first items in right side of Eqs. (8) and (9) are the union of all subgroups, and the second items are the intersections of any two subgroups. The subtraction of two items elimilates isomorphic configurations.

The permutation group is used to obtain the all permutation types of generalized kinematic pairs and generalized 


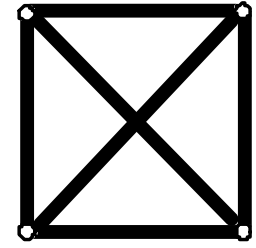

Figure 3. Planar 6-bar frame structure.

links. A permutation group of a set $A$ denoted as $S(A)$, is a set of permutations of $A$ that forms a group under composition of functions (Kurzweil and Stellmacher, 2004; Richard, 2009). It is the full permutation of elements in a group. Thus, the full-permutation vector groups of links and kinematic pairs are derived respectively as follows:

$\boldsymbol{G}=S\left(\boldsymbol{G}_{\mathrm{s}}\right)$

$\boldsymbol{Y}=S\left(\boldsymbol{Y}_{\mathrm{s}}\right)$.

\subsection{Configuration matrix groups of links and kinematic pairs}

First, the two mapping relationships are defined as follows:

1. $\mathbf{A}_{p}=J[G(p)]$, the operator $\boldsymbol{J}$ means the $p$ th vector of $G$ is placed at the main diagonal of matrix $\mathbf{A}_{p}$, and the others elements of matrix $\mathbf{A}_{p}$ are zeros.

2. $\mathbf{B}_{q}=H[Y(q)]$, the operator $\boldsymbol{H}$ means the $q$ th vector of $Y$ are placed the same location as the kinematic pairs of initial matrix, and the other elements of matrix $\mathbf{B}_{q}$ are zeros.

It can be noted that the dimension of matrixes $\mathrm{A}_{p}$ and $\mathrm{B}_{q}$ is the same as that of initial matrix. By the two mapping relationships, the full-permutation vector groups of links and kinematic pairs are transformed into the configuration matrix groups of links and kinematic pairs, respectively. They are $\mathbf{A} \mathbf{L}=\left\{\mathbf{A}_{1}, \cdots, \mathbf{A}_{p}, \cdots, \mathbf{A}_{v}\right\}$ and $\mathbf{B K}=\left\{\mathbf{B}_{1}, \cdots, \mathbf{B}_{q}, \cdots, \mathbf{B}_{w}\right\}$. The addition of $\mathbf{A}_{p}$ and $\mathbf{B}_{q}$ can create a configuration matrix, that is

$\mathbf{C}_{p q}=\mathbf{A}_{p}+\mathbf{B}_{q}(p=1, \cdots, v ; q=1, \cdots, w)$.

Then, the configuration matrix group is generated as follows.

$\mathbf{C}=\left\{\mathbf{C}_{11}, \cdots, \mathbf{C}_{1 q}, \cdots, \mathbf{C}_{p q}, \cdots, \mathbf{C}_{v w}\right\}$

It can be seen that an element of group $\mathbf{C}$ corresponds to a configuration matrix of a generalized deployable unit.

\subsection{Removing the unreasonable configurations and isomorphism detection}

The unreasonable configurations may exist in the configuration matrix group. In order to remove the unreasonable configurations, we propose some synthesis rules as follows.
1. The bars are replaced sequentially by flexible links, springs, or cables, respectively.

2. The rotational and cylindrical kinematic pairs are replaced by the preloaded hinges, flexible hinges, or fixed joints, respectively.

3. The fixed joint cannot connect two bars.

4. The preloaded hinge is composed of a torsional spring and a pair, which can only connect two bars.

5. The flexible hinge can connect two bars, two flexible links, a bar and a flexible link, respectively.

6. The connections between two springs, two cables, or a spring and a cable cannot exist.

The above synthesis rules can be performed based on the information of configuration matrix of generalized deployable units. In addition, Li et al. (2011) have proposed the method of the powers of the adjacency matrix for identifying the isomorphism of topological graphs and weighted multicolored graphs conveniently, correctly and uniquely. Thus the powers of configuration matrix are used to identify the isomorphism configurations of generalized deployable units.

\section{Configurations of 4 -link and 6-link generalized deployable units}

First, the proposed synthesis method is illustrated by the configuration generation of 4-link generalized deployable unit by taking the planar four-bar kinematic chain as the initial configuration.

The synthesis process is as follows:

1. The links generator is $a=[1,1,1,1]$, and the kinematic pairs generator is $b=[5,5,5,5]$.

2. The vector subgroups of generalized links can be obtained from Eqs. (4) and (5) as

$$
\begin{aligned}
& \boldsymbol{G}_{1}=\{[1,1,1,1],[2,1,1,1],[3,1,1,1],[4,1,1,1]\} \\
& \boldsymbol{G}_{2}=\{[1,1,1,1],[1,2,1,1],[1,3,1,1],[1,4,1,1], \\
& {[2,1,1,1],[2,2,1,1],[2,3,1,1],[2,4,1,1],} \\
& {[3,1,1,1],[3,2,1,1],[3,3,1,1],[3,4,1,1]} \\
& [4,1,1,1],[4,2,1,1],[4,3,1,1],[4,4,1,1]\} \\
& \ldots
\end{aligned}
$$

Meanwhile, the vector subgroups of kinematic pairs can be obtained from Eqs. (6) and (7) as

$$
\begin{aligned}
& \boldsymbol{Y}_{1}=\{[5,5,5,5],[7,5,5,5],[8,5,5,5],[9,5,5,5]\} \\
& \boldsymbol{Y}_{2}=\{[5,5,5,5],[5,7,5,5],[5,8,5,5],[5,9,5,5], \\
& {[7,5,5,5],[7,7,5,5],[7,8,5,5],[7,9,5,5],} \\
& {[8,5,5,5],[8,7,5,5],[8,8,5,5],[8,9,5,5],[9,5,5,5],}
\end{aligned}
$$




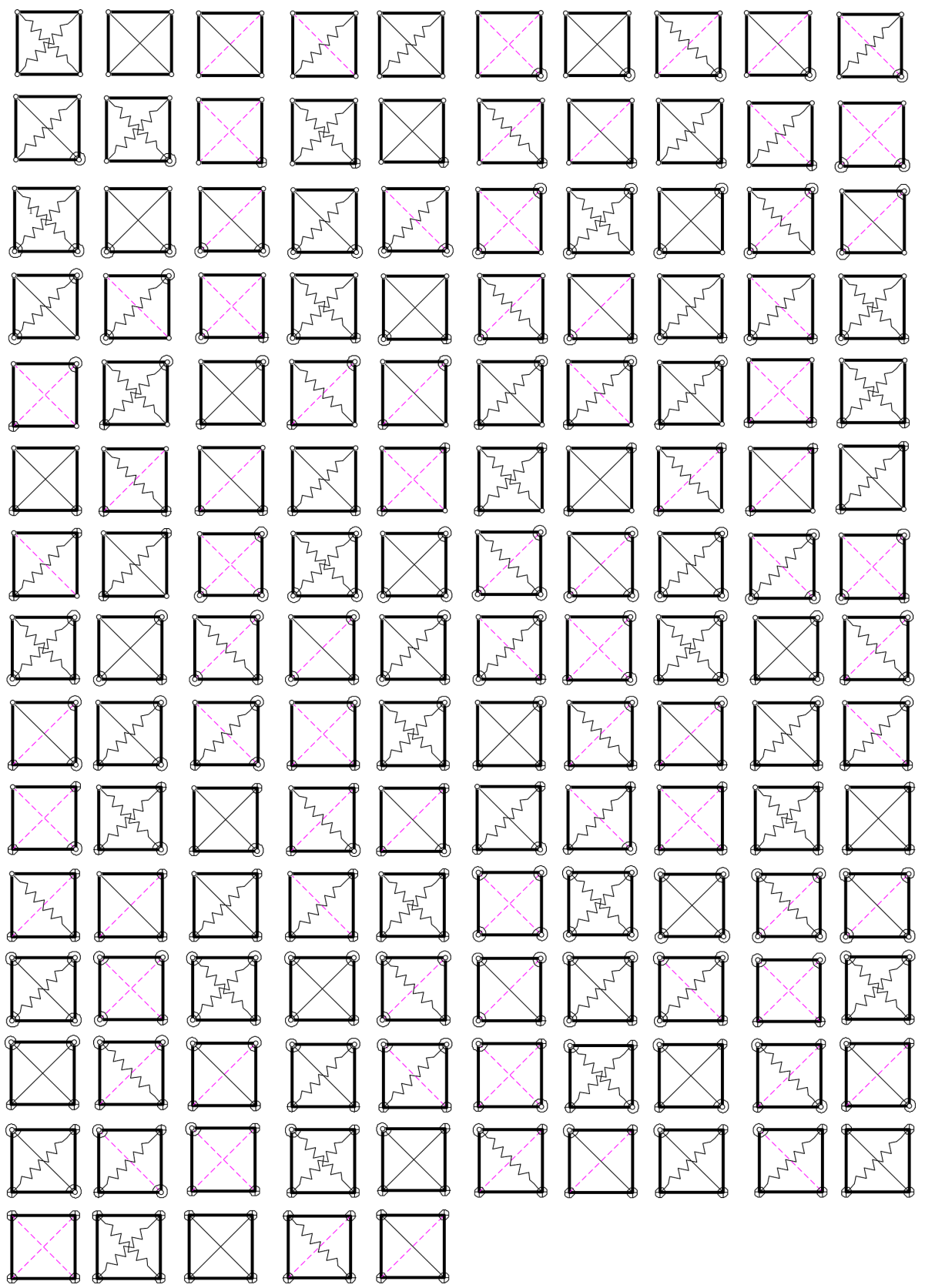

Figure 4. Configurations of 6-link generalized deployable unit with the rigid peripheral four links.

$[9,7,5,5],[9,8,5,5],[9,9,5,5]\}$

...

3. The isomorphic configurations of generalized links and kinematic pairs can be eliminated according to Eqs. (8) and (9), and the vector groups of generalized links and kinematic pairs are obtained as follows.

$$
\begin{aligned}
\boldsymbol{G}_{\mathrm{s}}= & \{[1,1,1,1],[2,1,1,1],[3,1,1,1],[4,1,1,1], \\
& {[1,2,1,1],[1,3,1,1],[1,4,1,1],[2,1,1,1], } \\
& {[2,2,1,1],[2,3,1,1],[2,4,1,1],[3,1,1,1], }
\end{aligned}
$$

$$
\begin{aligned}
& {[3,2,1,1],[3,3,1,1],[3,4,1,1], \cdots\} } \\
\boldsymbol{Y}_{\mathrm{S}}= & \{[5,5,5,5],[7,5,5,5],[8,5,5,5],[9,5,5,5], \\
& {[5,7,5,5],[5,8,5,5],[5,9,5,5], } \\
& {[7,7,5,5],[7,8,5,5],[7,9,5,5], } \\
& {[8,7,5,5],[8,8,5,5],[8,9,5,5], \cdots\} . }
\end{aligned}
$$

4. The configuration matrix groups of links and kinematic pairs are established respectively, as

$\mathbf{A L}=\left\{A_{1}, \cdots A_{l}, \cdots A_{m 1}\right\}$ 
Table 3. Configurations of 6-link generalized deployable unit.

\begin{tabular}{lc}
\hline Types of peripheral four links & $\begin{array}{c}\text { Number of } \\
\text { configurations }\end{array}$ \\
\hline Four rigid links & 145 \\
Three rigid links and a spring & 40 \\
Three rigid links and a cable & 40 \\
Two rigid links and two flexible links & 180 \\
Two rigid links, a flexible links and a spring & 99 \\
Two rigid links, a flexible link and a cable & 102 \\
A rigid link and three flexible links & 68 \\
A rigid link, two flexible links and a spring & 77 \\
A rigid link, two flexible link and a cable & 77 \\
A rigid link, a flexible link, a cable and a spring & 15 \\
Two rigid links and two cables & 6 \\
A rigid link, two cables and a flexible link & 6 \\
Four flexible links & 38 \\
Three flexible links and a spring & 19 \\
Two flexible links and two springs & 6 \\
Two flexible links, a cable and a spring & 6 \\
Two flexible links and two cables & 6 \\
\hline Total & 930 \\
\hline
\end{tabular}

$$
=\left\{\begin{array}{llllllll}
1 & 0 & 0 & 0 & 2 & 0 & 0 & 0 \\
0 & 1 & 0 & 0 & 0 & 1 & 0 & 0 \\
0 & 0 & 1 & 0 & 0 & 0 & 1 & 0 \\
0 & 0 & 0 & 1, & 0 & 0 & 0 & 1
\end{array}\right\}, \cdots
$$

$$
\begin{aligned}
& \mathbf{B K}=\left\{B_{1}, \cdots, B_{q}, \cdots, B_{w}\right\} \\
& =\left\{\begin{array}{llllllll}
0 & 5 & 0 & 5 & 0 & 7 & 0 & 5 \\
5 & 0 & 5 & 0 & 7 & 0 & 5 & 0 \\
0 & 5 & 0 & 5 & 0 & 5 & 0 & 5 \\
5 & 0 & 5 & 0, & 5 & 0 & 5 & 0
\end{array}\right\}
\end{aligned}
$$

5. The configuration matrix group of generalized deployable units is derived by Eqs. (12) and (13), as

$$
\begin{aligned}
& \mathbf{C}=\left\{C_{11}, \cdots, C_{1 q}, \cdots, C_{p q}, \cdots, C_{v w}\right\} \\
& =\left\{\begin{array}{llllllll}
1 & 5 & 0 & 5 & 1 & 7 & 0 & 5 \\
5 & 1 & 5 & 0 & 7 & 1 & 5 & 0 \\
0 & 5 & 1 & 5 & 0 & 5 & 1 & 5 \\
5 & 0 & 5 & 1, & 5 & 0 & 5 & 1
\end{array}\right\} .
\end{aligned}
$$

All the 186 configurations of 4-link generalized deployable unit are created, as shown in Fig. 2.

Then, the planar 6-bar frame structure, as shown in Fig. 3, is regarded as the initial configuration. All the 930 configurations of 6-link generalized deployable unit are created, as shown in Table 3 according to the types of peripheral four links. If the peripheral four links are rigid, the number of configurations is 145, as shown in Fig. 4. Some potential applications of synthesized configurations need to develop in the future. Obviously, the second configuration in Fig. 4 can be assembled into the deployable mechanism of Astromesh antenna (Thomson, 1999).

\section{Conclusions}

The paper proposes a configuration synthesis method of generalized deployable units composed of generalized links and generalized kinematic pairs based on the group theory. The permutation group of generalized kinematic pairs and generalized links are established and the topological configurations of generalized deployable units are generated. The configurations of generalized deployable units including four and six generalized links are created. The proposed method can also be applied to generate the other configurations of generalized deployable units, which will provide the enough configurations for the innovate design of large deployable mechanisms. The networking method of generalized deployable units will become keystone of research in the future.

Acknowledgements. The authors gratefully acknowledge the support of the National Natural Science Foundation of China (grant no. 51375360).

Edited by: X. Ding

Reviewed by: two anonymous referees

\section{References}

Chen, Y. and You, Z.: Two-fold symmetrical 6R foldable frame and its bifurcations, Int. J. Solids Struct., 46, 4504-4514, 2009.

Davies, T. H. and Crossley, F. E.: Structural analysis of plane linkages by Franke's condensed notation, J. Mechanisms, 1, 171183, 1966.

Ding, X. L., Yang, Y., and Dai, J. S.: Design and kinematic analysis of a novel prism deployable mechanism, Mech. Mach. Theory, 63, 35-49, 2013.

Gantes, C.: A design methodology for deployable structures, Ph.D. Thesis, Massachusetts Institute of Technology, 1991.

Kurzweil, H., Stellmacher, B.: The theory of finite groups: An introduction, Springer-Verlag, 1-92, 2004.

Li, T. J.: Deployable analysis and control of deployable space antenna, Aerosp. Sci. Technol., 18, 42-47, 2014.

Li, T. J., Cao, W. Q., and Yan, T. H.: Applications of graph theory in mechanism analysis, in: Emerging topics on differential geometry and graph theory, edited by: Lucas, B. and Francois, R., New York, Nova Science Publishers, 1-34, 2011.

Lu, S. N., Zlatanov, D., Ding, X. L., and Molfino, R.: A new family of deployable mechanisms based on the Hoekens linkage, Mech. Mach. Theory, 73, 130-153, 2014.

Lu, S. N., Zlatanov, D., Ding, X. L., Zoppi, M., and Guest, S. D.: A Network of Type III Bricard Linkages, ASME 2015 International Design Engineering Technical Conferences and Computers and Information in Engineering Conference, Boston, Massachusetts, USA, Paper No. DETC2015-47139, 2015.

Mruthyunjaya, T. S.: Kinematic structure of mechanisms revisited, Mech. Mach. Theory, 38, 279-320, 2003.

Pellegrino, S.: Deployable structures, Springer, 1-36, 2001.

Pinero, E. P.: Expandable space framing, Progressive Architecture, 43, 154-155, 1962. 
Richard, A. B.: Introductory combinatorics, 5th ed., Pearson, New York, 1-52, 2009.

Thomson, M. W.: The Astromesh deployable reflector, IEEE Antennas Prop., 3, 1516-1519, 1999.

Tibert, G.: Deployable tensegrity structures for space applications, Ph.D. Thesis, Sweden, Royal Institute of Technology, 2002.
Warnaar, D. B. and Chew, M.: Kinematic synthesis of deployablefoldable truss structure using graph theory, part 1: graph generation, J. Mech. Design, 117, 112-116, 1995a.

Warnaar, D. B. and Chew, M.: Kinematic synthesis of deployablefoldable truss structure using graph theory, part 2: generation of deployable truss module design concepts, J. Mech. Design, 117, 117-122, 1995b. 